\title{
Equation of state at finite density in two-flavor QCD with improved Wilson quarks
}

\author{
S. Aoki, ${ }^{a}{ }^{b}$ S. Ejiri, ${ }^{c}$ T. Hatsuda, ${ }^{d}$ N. Ishii, ${ }^{e}$ K. Kanaya $*{ }^{a}$ Y. Maezawa,${ }^{f}$ N. Ukita, ${ }^{e}$ and \\ T. Umeda ${ }^{a}$ (WHOT-QCD Collaboration) \\ ${ }^{a}$ Graduate School of Pure and Applied Sciences, Univ. of Tsukuba, Tsukuba 305-8571, Japan \\ ${ }^{b}$ RIKEN BNL Research Center, Brookhaven National Laboratory, Upton, New York 11973, USA \\ ${ }^{c}$ Physics Department, Brookhaven National Laboratory, Upton, New York 11973, USA \\ ${ }^{d}$ Department of Physics, Univ. of Tokyo, Tokyo 113-0033, Japan \\ ${ }^{e}$ Center for Computational Sciences, Univ. of Tsukuba, Tsukuba 305-8577, Japan \\ ${ }^{f}$ En'yo Laboratory, Nishina Accelerator Research Center, RIKEN, Wako 351-0198, Japan \\ E-mail: kanaya@ccs.tsukuba.ac.jp
}

\begin{abstract}
We study the equation of state in two-flavor QCD at finite temperature and density. Simulations are made with the RG-improved gluon action and the clover-improved Wilson quark action. Along the lines of constant physics for $m_{\mathrm{PS}} / m_{\mathrm{V}}=0.65$ and 0.80 , we compute the derivatives of the quark determinant with respect to the quark chemical potential $\mu_{q}$ up to the fourth order at $\mu_{q}=0$. We adopt several improvement techniques in the evaluation. We study thermodynamic quantities and quark number susceptibilities at finite $\mu_{q}$ using these derivatives. We find enhancement of the quark number susceptibility at finite $\mu_{q}$, in accordance with previous observations using staggered-type quarks. This suggests the existence of a nearby critical point.
\end{abstract}

The XXVI International Symposium on Lattice Field Theory

July 14-19 2008

Williamsburg, Virginia, USA

\footnotetext{
${ }^{*}$ Speaker.
} 

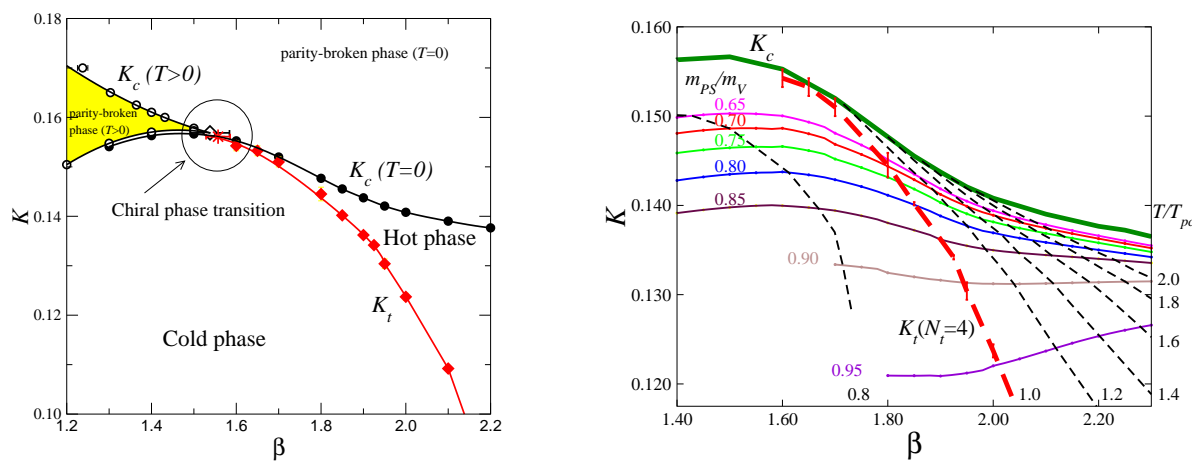

Figure 1: Phase diagram and lines of constant physics at $\mu=0$ for two-flavor QCD with improved Wilson quarks and Iwasaki glue at $N_{t}=4$ [3, 5 .

\section{Introduction}

Finite density QCD has been studied on the lattice mainly using staggered-type quarks [1]. However, because the expected $\mathrm{O}(4)$ universality of the deconfining transition in two-flavor QCD has not been confirmed with staggered-type quarks, the results may contain sizable lattice artifacts. Therefore, we need to crosscheck the results with different lattice actions. We study it with Wilsontype quarks, with which the $\mathrm{O}(4)$ scaling has been confirmed [2, 3].

Because Wilson-type quarks are numerically more intensive, we have to adopt/develop several improvement techniques. We apply a hybrid method of Taylor expansion and spectral reweighting, and develop a couple of improvement tricks.

\section{Formulation}

We extend the study of two-flavor QCD by the CP-PACS Collaboration at vanishing chemical potential $\mu_{q}=0$ [3] to finite densities. Preliminary reports of this study have been presented at Lattice 2006 and 2007 conferences [4].

We adopt the clover-improved Wilson quark action and the RG-improved Iwasaki gauge action defined by $S=S_{g}+S_{q}$ with

$$
S_{g}=-\beta \sum_{x}\left(c_{0} \sum_{\mu<\nu ; \mu, v=1}^{4} W_{\mu \nu}^{1 \times 1}(x)+c_{1} \sum_{\mu \neq \nu ; \mu, v=1}^{4} W_{\mu \nu}^{1 \times 2}(x)\right), \quad S_{q}=\sum_{f=1}^{2} \sum_{x, y} \bar{q}_{x}^{f} M_{x, y} q_{y}^{f},
$$

where $\beta=6 / g^{2}, c_{1}=-0.331, c_{0}=1-8 c_{1}$, and

$$
\begin{aligned}
M_{x, y}= & \delta_{x y}-K \sum_{i=1}^{3}\left\{\left(1-\gamma_{i}\right) U_{x, i} \delta_{x+\hat{i}, y}+\left(1+\gamma_{i}\right) U_{x, i}^{\dagger} \delta_{x, y+\hat{i}}\right\} \\
& -K\left\{e^{\mu}\left(1-\gamma_{4}\right) U_{x, 4} \delta_{x+\hat{4}, y}+e^{-\mu}\left(1+\gamma_{4}\right) U_{x, 4}^{\dagger} \delta_{x, y+\hat{4}}\right\}-\delta_{x y} c_{S W} K \sum_{\mu<v} \sigma_{\mu v} F_{\mu v} .
\end{aligned}
$$

where $\mu \equiv \mu_{q} a$ and $F_{\mu \nu}$ is the lattice field strength in terms of the standard clover-shaped combination of gauge links. For the clover coefficient $c_{S W}$, we adopt a mean field value using $W^{1 \times 1}$ calculated in the one-loop perturbation theory: $c_{S W}=\left(W^{1 \times 1}\right)^{-3 / 4}=\left(1-0.8412 \beta^{-1}\right)^{-3 / 4}[3]$. 
The lattice size is $N_{s}^{3} \times N_{t}=N_{\text {site }}=16^{3} \times 4$. We carry out simulations along the lines of constant physics (LOCs) for $m_{P S} / m_{V}=0.65$ and 0.80 at $\mu_{q}=0$ [5] shown in Fig 1] For definiteness, we refer the pseudocritical temperature $T_{p c}$ at $\mu=0$ as $T_{0}$ in the followings. Runs are carried out in the range $\beta=1.50-2.40$ at thirteen values of $T / T_{0} \sim 0.82-4.0$ for $m_{\mathrm{PS}} / m_{\mathrm{V}}=0.65$ and twelve values of $T / T_{0} \sim 0.76-3.0$ for $m_{\mathrm{PS}} / m_{\mathrm{V}}=0.80$. The number of trajectories for each run after thermalization is 5000-6000. We measure physical quantities at every 10 trajectories.

\section{Taylor expansion up to $O\left(\mu_{q}^{4}\right)$}

We expand the grand canonical potential $\omega=p / T^{4}$ in terms of $\mu_{q} / T$ at $\mu_{q}=0$ :

$$
\omega=\frac{1}{V T^{3}} \ln \mathscr{Z}=\sum_{n=0}^{\infty} c_{n}(T)\left(\frac{\mu_{q}}{T}\right)^{n}, \quad c_{n}(T)=\left.\frac{1}{n !} \frac{N_{t}^{3}}{N_{s}^{3}} \frac{\partial^{n} \ln \mathscr{Z}}{\partial\left(\mu_{q} / T\right)^{n}}\right|_{\mu_{q}=0},
$$

where $V=\left(N_{s} a\right)^{3}$ is the system volume and $\mathscr{Z}$ is the partition function. We expand up to $\left(\mu_{q} / T\right)^{4}$. The relevant coefficients are given by

$$
\begin{aligned}
& c_{2}=\frac{N_{t}}{2 N_{s}^{3}} \mathscr{A}_{2}, \quad c_{4}=\frac{1}{4 ! N_{s}^{3} N_{t}}\left(\mathscr{A}_{4}-3 \mathscr{A}_{2}^{2}\right), \\
& \mathscr{A}_{2}=\left\langle\mathscr{D}_{2}\right\rangle+\left\langle\mathscr{D}_{1}^{2}\right\rangle, \quad \mathscr{A}_{4}=\left\langle\mathscr{D}_{4}\right\rangle+4\left\langle\mathscr{D}_{3} \mathscr{D}_{1}\right\rangle+3\left\langle\mathscr{D}_{2}^{2}\right\rangle+6\left\langle\mathscr{D}_{2} \mathscr{D}_{1}^{2}\right\rangle+\left\langle\mathscr{D}_{1}^{4}\right\rangle,
\end{aligned}
$$

where $\mathscr{D}_{n}=N_{f}\left(\frac{\partial}{\partial \mu}\right)^{n} \ln \operatorname{det} M$ is calculated as

$$
\mathscr{D}_{1}=N_{f} \operatorname{tr}\left(M^{-1} \frac{\partial M}{\partial \mu}\right), \quad \mathscr{D}_{2}=N_{f}\left[\operatorname{tr}\left(M^{-1} \frac{\partial^{2} M}{\partial \mu^{2}}\right)-\operatorname{tr}\left(M^{-1} \frac{\partial M}{\partial \mu} M^{-1} \frac{\partial M}{\partial \mu}\right)\right], \quad \text { etc. }
$$

We calculate similar coefficients for the expansion in terms of the isospin chemical potential $\mu_{I}$ too.

We evaluate the traces with the random noise method. In the evaluation, we apply the following two improvements: (i) Because the elements off-diagonal in color and spin indices are not suppressed by $|x-y|$ with Wilson-type quarks, the number of the same-magnitude off-diagonal elements in the quark matrix is 11 times larger than the diagonal one. This is different from the case of staggered-type quarks, in which off-diagonal elements in spin indices are slightly suppressed by the spatial offset. Because a large number of noises is required to pick up a signal from data with $S / N=1 / 11$, we decide not to apply the noise method for color and spin indices and generate noise vectors only for spatial indices, i.e. we repeat the inversion of $M$ for each color and spin indices. (ii) We find that the dominant errors are from terms containing $\mathscr{D}_{1}$. Therefore, we adopt 10-40 times more noise vectors for $\mathscr{D}_{1}$. while we generate only 10 noise vectors for other traces.

Results for the pressure difference $\Delta p=p\left(\mu_{q}\right)-p(0)$, quark number density $\left(n_{q}\right)$, and quark number as well as isospin susceptibilities $\left(\chi_{q}\right.$ and $\chi_{I}$, respectively) are shown in Fig 2 for $m_{\mathrm{PS}} / m_{\mathrm{V}}=$ 0.65 . We observe larger enhancement in the quark number fluctuations near $T_{0}$ with increasing $\mu_{q}$, however such an enhancement around $T_{0}$ is not shown in the isospin fluctuations. Because the statistical errors in $n / T^{3}$ and $\chi_{q} / T^{2}$ are large, further studies are needed increasing the statistics for more precise arguments with this approach. 

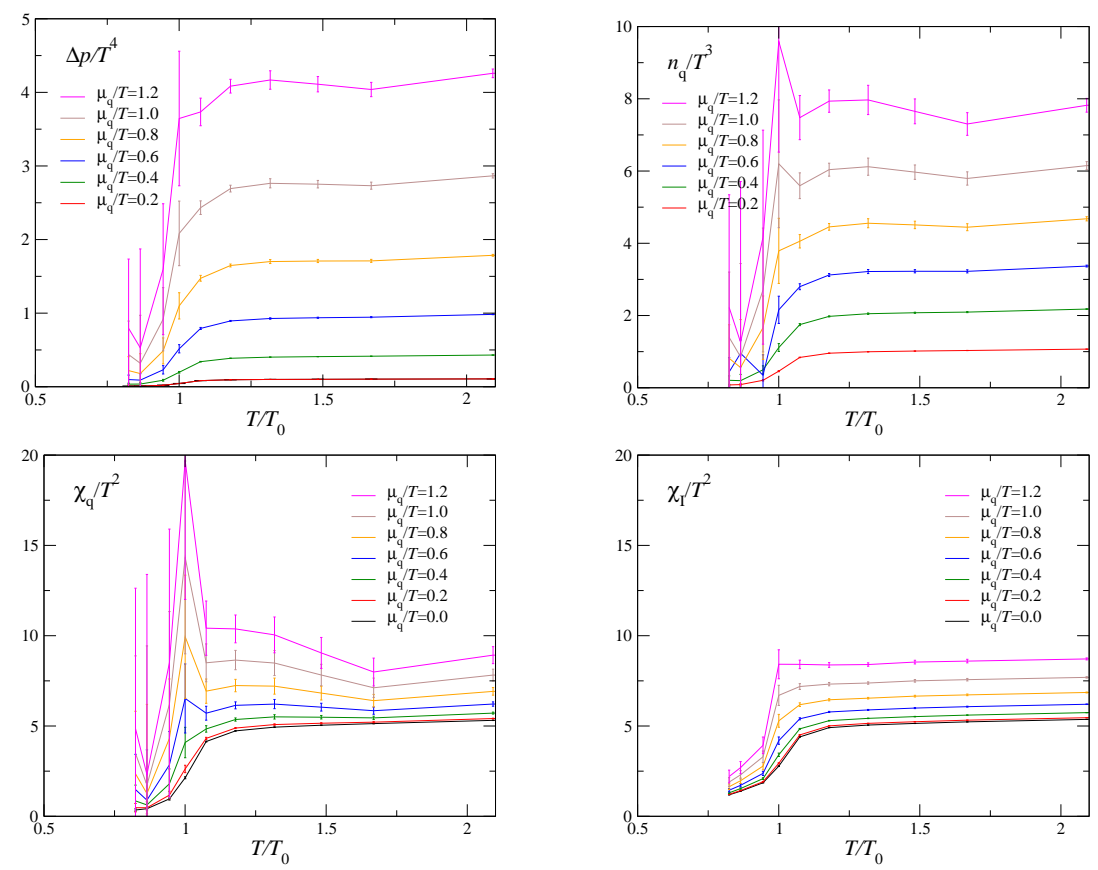

Figure 2: Results of the Taylor expansion method up to the fourth order of chemical potentials for $m_{\mathrm{PS}} / m_{\mathrm{V}}=$ 0.65. $T_{0}$ is the pseudocritical temperature at $\mu=0$ for the same LOC.

\section{Improvement: A Hybrid Method}

In the previous section, the Taylor expansion is limited up to the fourth order of the chemical potential, because the evaluation of $\mathscr{D}_{n}$ with $n>4$ is compuationally demanding. To improve the calculation, we need to estimate $c_{n}$ at larger $n$. Here, we note that $\mathscr{D}_{n}=0$ at $n>4$ for the free quark case. Therefore, at high temperatures, we may approximate $\mathscr{D}_{n}=0$ for $n>4$ in the evaluation of $c_{n}$ at $n>4$. This approximation corresponds to a hybrid reweighting method in which the grand canonical potential is approximated by a truncated Taylor expansion

$$
\omega(T, \mu) \approx \frac{1}{V T^{3}} \ln \mathscr{Z}(T, 0)+\frac{1}{V T^{3}} \ln \left\langle\exp \left[\sum_{n=1}^{N_{\max }} \mathscr{D}_{n} \mu^{n}\right]\right\rangle_{(\mu=0)},
$$

with $N_{\max }=4$. Here, $\langle\cdots\rangle_{(\mu=0)}$ is the average over configurations at $\mu=0$. We then have

$$
\mathscr{Z}(T, \mu) \approx \mathscr{Z}(T, 0)\left\langle e^{F(\mu)} e^{i \theta(\mu)}\right\rangle_{(\mu=0)}
$$

with

$$
\begin{aligned}
& \theta(\mu) \equiv N_{\mathrm{f}} \operatorname{Im}[\ln \operatorname{det} M(\mu)] \approx \operatorname{Im} \mathscr{D}_{1} \mu+\frac{1}{3 !} \operatorname{Im} \mathscr{D}_{3} \mu^{3}, \\
& F(\mu) \equiv N_{\mathrm{f}} \operatorname{Re}\left[\ln \left(\frac{\operatorname{det} M(\mu)}{\operatorname{det} M(0)}\right)\right] \approx \frac{1}{2 !} \operatorname{Re} \mathscr{D}_{2} \mu^{2}+\frac{1}{4 !} \operatorname{Re} \mathscr{D}_{4} \mu^{4} .
\end{aligned}
$$

This kind of hybrid method was first tested in [6] with two flavors of staggered quarks. In that study, it turned out to be difficult to control statistical errors at $\mu_{q}$ larger than $O(T)$. This is due to the sign problem at finite $\mu_{q}$. 

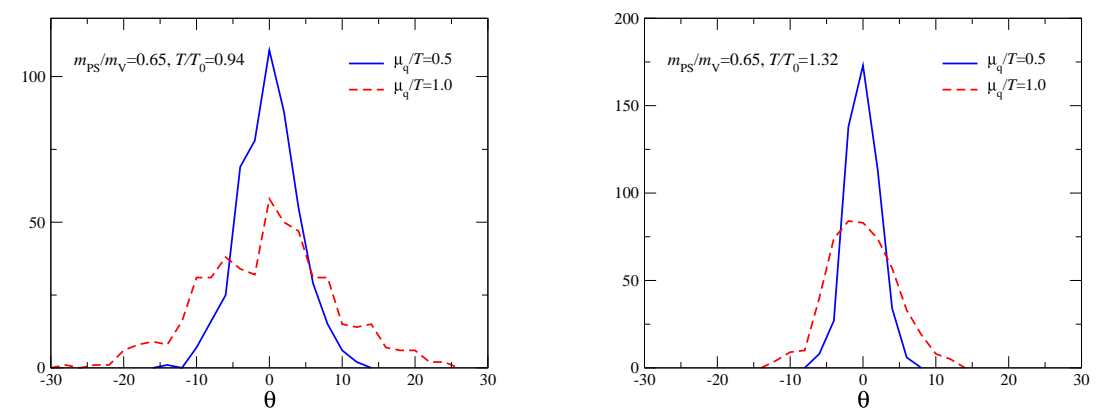

Figure 3: Typical $\theta$-distribution of two-flavor QCD at $\mu_{q}=0$ for $m_{\mathrm{PS}} / m_{\mathrm{V}}=0.65$.
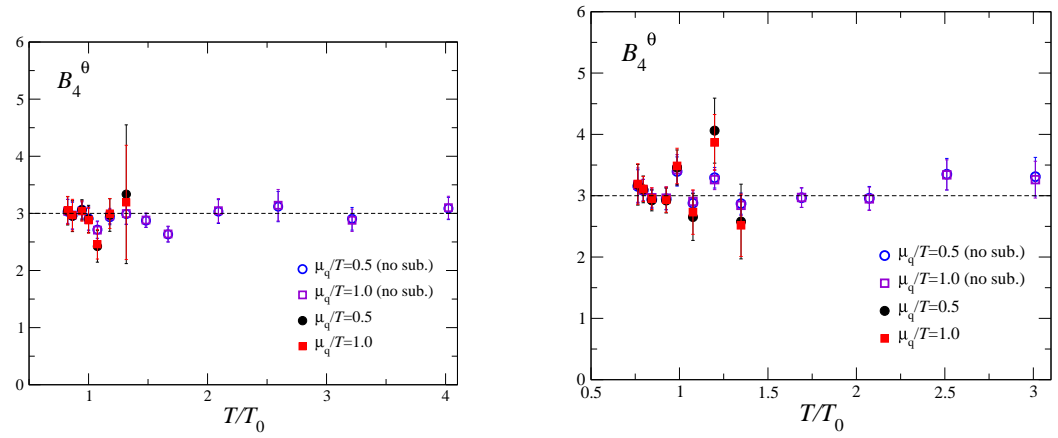

Figure 4: Binder cumulant test of $\theta$-distribution for $m_{\mathrm{PS}} / m_{\mathrm{V}}=0.65$ (left) and 0.80 (right). Data labeled as "no sub." are the results of approximate estimation of $\theta^{2}$ and $\theta^{4}$ without subtracting the contribution of the same noise vectors. Data without "no sub." are the correct estimates.

In a previous study with staggered quarks, SE noted that the $\theta$-distribution at $\mu=0$ is well described by a Gaussian form, and showed that this fact can be used to carry out the $\theta$-averaging with small errors [7]. Furthermore, we can argue that, because $\theta=\mathscr{O}(\mu)$ at small $\mu$, deviations from the Gaussian distribution do not affect the terms up to $\mu^{4}[8,9$. We find that our data are also well Gaussian (see Fig 3 for typical examples). A convenient test of Gaussian distributions is provided by the Binder cumulant $B_{4}^{\theta}=\left\langle\theta^{4}\right\rangle /\left\langle\theta^{2}\right\rangle^{2}$. If the distribution is Gaussian, we expect $B_{4}^{\theta}=3$. In Fig 4, we show that all of our distributions are approximately consistent with $B_{4}^{\theta}=3$.

With Gaussian $\theta$-distribution, we can carry out the $\theta$-averaging, and the task is reduced to evaluate

$$
\mathscr{Z}(T, \mu) \approx \mathscr{Z}(T, 0)\left\langle e^{F} e^{-\frac{1}{2}\left\langle\theta^{2}\right\rangle_{F}}\right\rangle_{(\mu=0)},
$$

with $\left\langle\theta^{2}\right\rangle_{F}$ the mean $\theta^{2}$ for given fixed $F$.

The remaining $F$-averaging is again challenging because the factor $e^{F(\mu)}$ can easily shift the central contribution for the average to a statistically poor region of $F$. At small $\mu$, this problem can be largely resolved by shifting $\beta$ with $\mu$ such that the fluctuation in $e^{F(\mu)}$ is compensated by that in the gauge action, because $F$ is sensitively correlated with the gauge action $S_{g}$. More concretely, we adopt the reweighting method to shift $\beta$ and calculate an optimal $\beta$ by minimizing the fluctuation

$$
\left\langle\left(e^{F} e^{-\frac{1}{2}\left\langle\theta^{2}\right\rangle_{F}} e^{6 N_{\text {site }}\left(\beta-\beta_{0}\right) P}-\left\langle e^{F} e^{-\frac{1}{2}\left\langle\theta^{2}\right\rangle_{F}} e^{6 N_{\text {site }}\left(\beta-\beta_{0}\right) P}\right\rangle\right)^{2}\right\rangle
$$



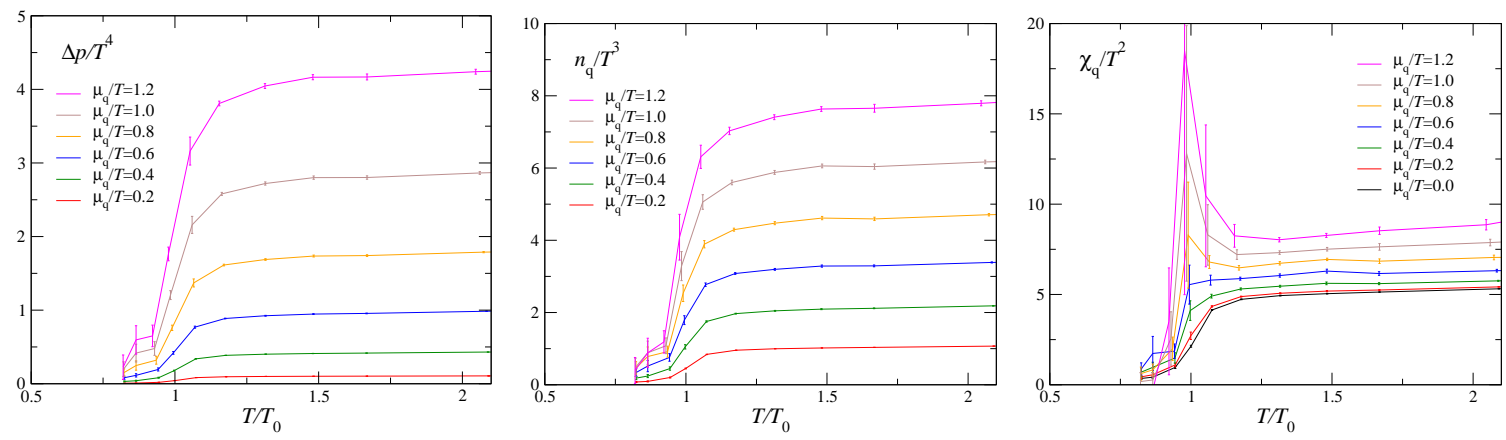

Figure 5: Results of EOS using the hybrid method for $m_{\mathrm{PS}} / m_{\mathrm{V}}=0.65$.
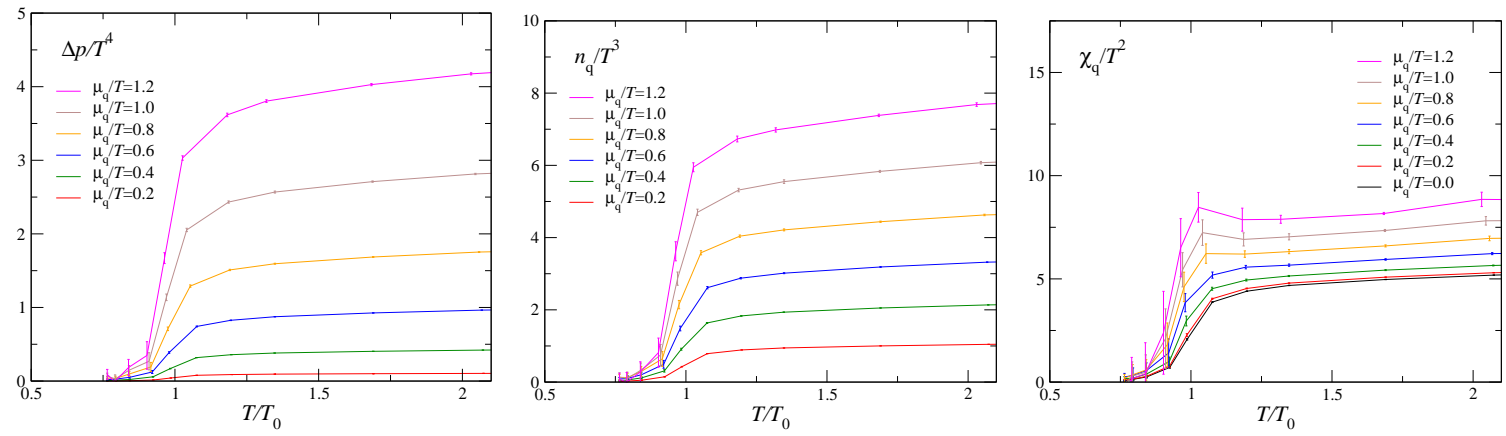

Figure 6: Results of EOS using the hybrid method for $m_{\mathrm{PS}} / m_{\mathrm{V}}=0.80$.

where $P=S_{g} /\left(6 N_{\text {site }} \beta\right)$ is the generalized plaquette. The shifts in $\beta$ turn out to be less than about 0.03 in our study. Since these are negligible in Fig 1 we disregard the resulting small deviation from the line of constant physics, and simply translate the shifts in $\beta$ to shifts in $T$ for the final plots.

Our results of EOS with these improvements are summarized in Figs 5 and 6 for $m_{\mathrm{PS}} / m_{\mathrm{V}}=$ 0.65 and 0.80 , respectively. Here, we calculate the quark number density and its susceptibility by numerical differentiations of the grand canonical potential using the following thermodynamic formulae:

$$
\frac{n_{q}}{T^{3}}=\frac{N_{t}^{3}}{N_{s}^{3}} \frac{\partial(\ln \mathscr{Z})}{\partial\left(\mu_{q} / T\right)}, \quad \frac{\chi_{q}}{T^{2}}=\frac{N_{t}^{3}}{N_{s}^{3}} \frac{\partial^{2}(\ln \mathscr{Z})}{\partial\left(\mu_{q} / T\right)^{2}} .
$$

We find that, in spite of the fact that the simulations at different $T / T_{0}$ are independent, the resulting EOS shown in these figures has smooth $T$ - and $\mu_{q}$-dependence which is in accordance with theoretical expectations. Therefore, we think that the assumption $\mathscr{D}_{n}=0$ at $n>4$ is well satisfied down to $T$ close to the transition temperature. Furthermore, we find that the statistical fluctuations are much reduced over the results of the previous section (Fig,2). This is due to the improvements including the Gaussian method for $\theta$-averaging and the $\beta$-reweighting for $F$-averaging. The hybrid method with improvements provides us with a tractable way to calculate EOS with Wilson-type quarks.

Similar to the case of the previous section, the quark number susceptibility for $m_{\mathrm{PS}} / m_{\mathrm{V}}=0.65$ show enhancement near $T_{0}$ with increasing $\mu_{q}$, although the statistical errors are not quite small yet. On the other hand, the quark number susceptibility for $m_{\mathrm{PS}} / m_{\mathrm{V}}=0.80$ shown in Fig 6 do not show 
rapid enhancement with $\mu_{q}$. This may be in part explained by the expectation that the critical point locates at larger $\mu_{q}$ because the quark mass is larger than that for $m_{\mathrm{PS}} / m_{\mathrm{V}}=0.65$. The mild enhancement shown in Fig 6 may be suggesting that, at this quark mass, the critical point does not locate inside the applicability range in $\mu_{q}$ with our $\mathscr{O}\left(\mu_{q}^{4}\right)$ calculation. Further studies with increased statistics around $T_{0}$ are needed for more definite conclusions.

\section{Conclusions}

We have carried out the first calculation of the equation of state at non-zero densities with two flavors of improved Wilson quarks. Statistical fluctuations of physical observables at finite density are much severer with Wilson-type quarks than with staggered-type quarks. To tame the problem, we combined and developed several improvement techniques.

With these improvements, we found that the peak height of the quark number fluctuation at the pseudo-critical temperature increases as $\mu_{q}$ increases. In contrast, isospin susceptibilities show no sharp peaks at the pseudo-critical temperature. These results agree with previous observations by the Bielefeld-Swansea Collaboration using staggered-type quarks, and suggest that a critical point exists at finite $\mu_{q}$, which is expected to locate at the end point of a first order transition line between confining and deconfining phases in the coupling parameter space of $T$ and $\mu_{q}$. Details of our study will be described in [9].

This work is in part supported by Grants-in-Aid of the Japanese Ministry of Education, Culture, Sports, Science and Technology (Nos. 17340066, 18540253, 19549001 and 20340047). SE is supported by U.S. Department of Energy (DE-AC02-98CH10886). Numerical calculations were performed on supercomputers at KEK by the Large Scale Simulation Program Nos. 06-19, 07-18, 08-10, at CCS, Univ. of Tsukuba, and at ACCC, Univ. of Tsukuba.

\section{References}

[1] S. Ejiri, in these proceedings, $\mathrm{POS} \quad$ (LATTICE 2008) 002

[2] Y. Iwasaki, K. Kanaya, S. Kaya and T. Yoshié, Phys. Rev. Lett. 78 (1997) 179.

[3] S. Aoki, et al., (CP-PACS Collaboration), Phys. Rev. D 63 (2000) 034502; ibid. 64 (2001) 074510.

[4] S. Ejiri, et al., (WHOT-QCD Collaboration), POS (LATTICE 2006) 132, Y. Maezawa, et al., (WHOT-QCD Collaboration), POS (LATTICE 2007) 207

[5] Y. Maezawa, et al., (WHOT-QCD Collaboration), Phys. Rev. D 75 (2007) 074501.

[6] C.R. Allton, et al., (Bielefelt-Swansea Collaboration), Phys. Rev. D 71 (2005) 054508.

[7] S. Ejiri, Phys. Rev. D 77 (2008) 014508.

[8] S. Ejiri, Phys. Rev. D, to be published, [ArXiv:hep-lat/0804.3227].

[9] S. Aoki, S. Ejiri, T. Hatsuda, N. Ishii, K. Kanaya, Y. Maezawa, N. Ukita, and T. Umeda (WHOT-QCD Collaboration), in preparation. 